\title{
735 IDENTIFICATION OF A SMALL MOLECULE THAT PREVENTS T CELL EXHAUSTION USING MACHINE LEARNING ALGORITHMS PAIRED WITH HIGH- RESOLUTION SINGLE CELL RNASEQ
}

Isabelle Le Mercier*, Sunny Sun, Dongmei Xiao, Laura Isacco, Daniel Treacy, Emilie Artru, Scott Steelman, John Bradley, Alex Wolf, Morag Stewart, Effie Tozzo. Cellarity, Cambridge, MA, United States

Background $\mathrm{T}$ cell responses are tightly regulated and require a constant balance of signals during the different stages of their activation, expansion, and differentiation. As a result of chronic antigen exposure, $\mathrm{T}$ cells become exhausted in solid tumors, preventing them from controlling tumor growth.

Methods We identified a transcriptional signature associated with $\mathrm{T}$ cell exhaustion in patients with melanoma and used our proprietary machine learning algorithms to predict molecules that would prevent $\mathrm{T}$ cell exhaustion and improve $\mathrm{T}$ cell function. Among the predictions, an orally available small molecule, Compound A, was highly predicted.

Results Compound A was tested in an in vitro T cell Exhaustion assay and shown to prevent loss of proliferation and expression of immune checkpoint receptors. Transcriptionally, Compound A-treated cells looked indistinguishable from conventionally expanded, non-exhausted $\mathrm{T}$ cells. However, when assessed in a classical $\mathrm{T}$ cell activation assay, Compound $\mathrm{A}$ demonstrated dose dependent activity. At low dose, Compound A was immuno-stimulatory, allowing cells to divide further by preventing activation induced cell death. At higher doses, Compound A demonstrated immuno-suppressive activity preventing early CD69 upregulation and T cell proliferation. All together, these observations suggest that Compound A prevented exhaustion with a mechanism of action involving TCR signaling inhibition. While cessation of TCR signaling or rest has been recently associated with improved CAR-T efficacy by preventing or reversing exhaustion during the in vitro manufacturing phase, it is unclear if that mechanism would translate in vivo.Compound A was evaluated in the CT26 and MC38 syngeneic mouse models alongside anti-PD1. At low dose Compound A closely recapitulated anti-PD1 mediated cell behavior changes by scRNA-seq and flow cytometry in CT26 mice. At high dose, Compound A led to the accumulation of naive cells in the tumor microenvironment (TME) confirming the proposed mechanism of action. Low dose treatment was ineffective in MC38 mouse model but a pulsed treatment at high dose also recapitulated anti-PD1 activity in most animals. Importantly, we identified a new $\mathrm{T}$ cell population responding to anti-PD1 that was particularly increased in the MC38 mouse model; Compound A treatment also impacted this population.

Conclusions These data confirm that mild TCR inhibition either suboptimal or fractionated can prevent exhaustion in vivo. However, this approach has a very limited window of activity between immuno-modulatory and immuno-suppressive effects, thereby limiting potential clinical benefit. Finally, these results demonstrate that our approach and platform was able to predict molecules that would prevent $\mathrm{T}$ cell exhaustion in vivo.

http://dx.doi.org/10.1136/jitc-2021-SITC2021.735 